\title{
Morphology of metallic foams synthetized by plasma electrolysis deposition
}

\author{
Julien Pinot ${ }^{1,2,3,}{ }^{*}$, Ronan Botrel ${ }^{1,3}$, Frederic Durut ${ }^{1,3}$, Ludovic Reverdy ${ }^{1}$, Laurent Pescayre ${ }^{1}$, and Vincent Vignal ${ }^{2,3}$ \\ ${ }^{1}$ Commissariat à l'Energie Atomique et aux Energies Alternatives, DAM, Valduc, 21120 Is sur Tille, France \\ 2 Laboratoire Interdisciplinaire Carnot de Bourgogne, UMR 6303 CNRS-Université de Bourgogne, BP 47870, 21078 Dijon, France \\ ${ }^{3}$ Laboratoire Interactions Matériau-Procédé-Environnement, LRC LIMPE, n VA-11-02, Dijon, France
}

Received: 19 October 2021 / Received in final form: 2 December 2021 / Accepted: 6 December 2021

\begin{abstract}
The aim of our work is to understand the mechanism governing the growth of metallic foams synthetized by plasma electrolysis deposition. This paper reports the influence of the applied voltage on the morphology and microstructure of copper and gold foams. The evolution of strands morphology and size is investigated by field emission scanning electronic microscopy (FE-SEM). The role of the voltage in the growth of metallic foams is then discussed. Finally, the crystalline structure of the strands is determined by transmission electronic microscopy (TEM) and selected area electron diffraction.
\end{abstract}

\section{Introduction}

In the vicinity of laser target experiments for plasma physics studies, the energy transfer from the laser impulse to the electrons and ions in the plasma is an important factor to the success of the experiments and near critical plasmas conditions exhibit a better efficiency for the energy transfer than denser plasmas [1]. One way to produce near critical plasma is through the interaction of a very intense laser beam $\left(I>10^{18} \mathrm{~W} / \mathrm{cm}^{2}\right)$ with a metallic foam.

Metallic foams are defined as three-dimensional porous materials composed of interconnected metallic particles or strands. This class of materials has been studied and developed for their unique properties due to their porous structure and their low-density compared to the bulk metal. Metallic foams are used in aeronautics and construction fields for their lightweight and shocks or acoustic waves absorption properties [2]. Others use their high specific area and conductivity for batteries, supercapacitors, chemical catalysis as well as chemical sensors [3]. Multiples processes are referenced for manufacturing metallic foams such as gas injection in liquid state metal [4], metallic powder compaction [5] or electrochemical deposition on organic foam [6]. These processes lead to metallic foams with an extensive range of density and porosity.

However, to be compatible with laser experiments, metallic foams must have a thin structure, a small pore size $(<1 \mu \mathrm{m})$, an apparent density under $1 \mathrm{~g} / \mathrm{cm}^{3}$ and a high purity $(>95 \%)$. Such metallic foams can't be obtained by previously described techniques. Thus laser target fabricants had to develop their own process to manufacture compatible metallic foams [7-9]. After ten years of research, the CEA patented a process for synthesizing

\footnotetext{
* e-mail: jpinot.pro@gmail.com
}

nanostructured metallic foams. This process is based on plasma electrolysis deposition (PED) or contact glow discharge electrolysis (CGDE): a hybrid technique of conventional electrolysis and plasma processing [10].

Our technique consists in creating and stabilizing a gas sheath around a cathode immerged in an aqueous electrolyte containing a metallic salt. Beyond a critical voltage, we observe the formation of electric sparks propagating from the cathode to the gas/liquid interface. Then upon contact of the sparks with the solution, we can observe the formation of thin metallic strands. The metallic strands continue to grow as long as the plasma regime is maintained. A few millimeters wide metallic foam is obtained in less than ten seconds. This extremely fast process is also very versatile. This way, we can synthesize pure metal foams $(\mathrm{Cu}, \mathrm{Zn}, \mathrm{Pt}, \mathrm{Au}$, etc.) or alloys $(\mathrm{AuCu}$, CuZn, etc.) according to the metal ions in the electrolyte. The aim of our work is to understand the phenomenon which governs the growth of our metallic foams in order to control the synthesis process. We have shown in a previous paper [11] that the composition of the electrolytic solution greatly influences the foam growth and the shape of the strands. In the present paper we investigate more closely the role of the applied voltage in the synthesis process by conducting FE-SEM and TEM investigations on the foam microstructure and on the morphology of the strands.

\section{Experimental setup and characterization techniques}

Our experimental setup (Fig. 1) consists in immersing two electrodes in a crystallizing dish containing $100 \mathrm{~mL}$ of the electrolytic solution. The anode is a platinum grid and the cathode is made of a tungsten cylinder about $1 \mathrm{~mm}$ in diameter with a spherical tip. The distance between the 


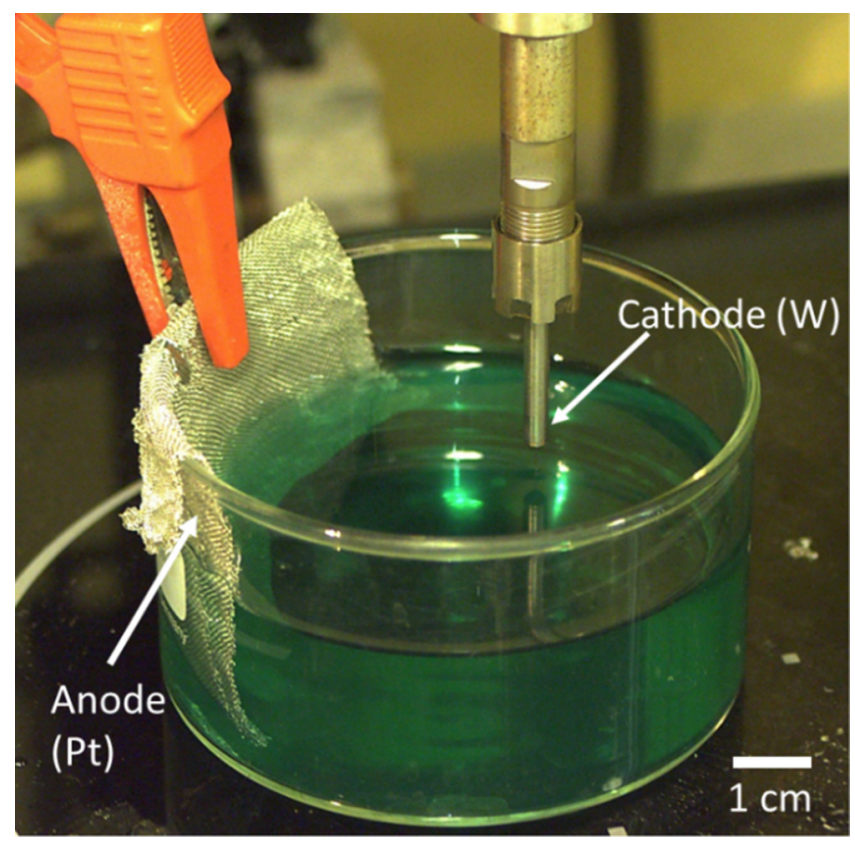

Fig. 1. Experimental setup for the synthesis of metallic foams.

electrodes is around $5 \mathrm{~cm}$. The aqueous electrolyte is composed of diluted hydrochloric acid $(\mathrm{HCl}, 37 \%$ Merck $-1 \mathrm{~mol} / \mathrm{L})$, and a metallic salt $(0.1 \mathrm{~mol} / \mathrm{L})$. The metallic salts used in this paper are either copper (II) chloride dihydrate $\left(\mathrm{CuCl}_{2}, 2 \mathrm{H}_{2} \mathrm{O}\right.$, Merck) or tetrachloroauric acid ( $\mathrm{HAuCl}_{4}$, Heraeus). The voltage current supply is provided by a PSI-9080 60DT electrical generator (Electro-Automatik). The applied voltage for the foam synthesis ranges between 25 and $60 \mathrm{~V}$ with current intensity up to $5 \mathrm{~A}$. The pictures of copper and gold foams are presented in Figure 2.

Powder X-ray diffraction was achieved on a Brucker D8 Discover diffractometer. Due to the structure of the foams, nearly 30 foams had to be compressed in order to obtain a significant signal.

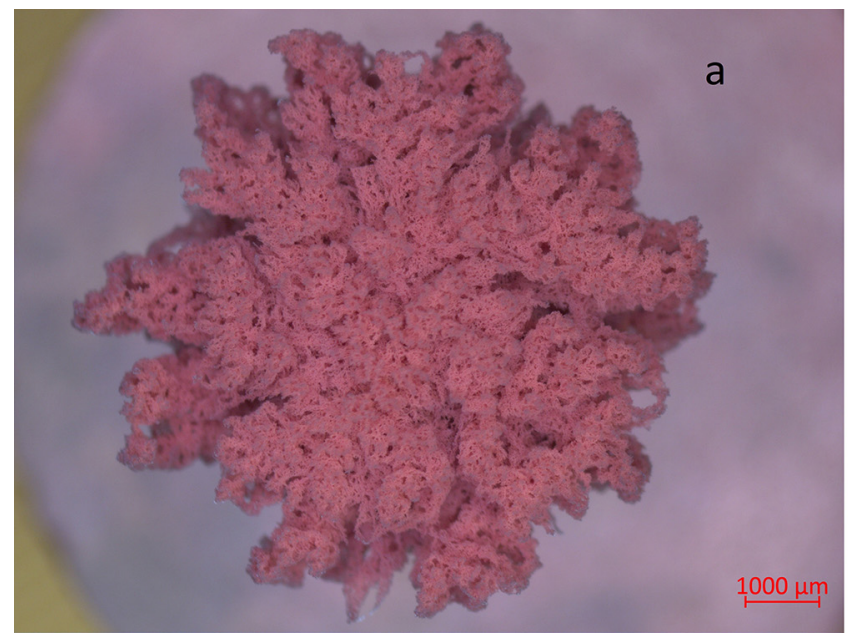

The foam structure and the strands morphology were characterized by field emission scanning electron microscopy (FE-SEM Leo Carl Zeiss 1525). Transmission electron microscopy (TEM) investigations were carried out to determine the crystalline structure of the foam strands using a JEM-ARM 200F Cold FEG TEM/STEM operating at $200 \mathrm{kV}$ and equipped with a spherical aberration (Cs) probe and image correctors (point resolution $0.12 \mathrm{~nm}$ in TEM mode and $0.078 \mathrm{~nm}$ in STEM mode). The TEM samples were prepared by ultra-sonicating the foam immerged in ethanol in order to detach and disperse the strands.

\section{Results and discussions}

\subsection{Evolution of the morphology of the strands with applied voltage}

The FE-SEM observations show that the foam structure varies greatly with the synthesis voltage. Figure 3 presents the evolution of the strands morphology with the applied voltage for copper foams.

From 25 to $30 \mathrm{~V}$, we observe thin $(50-100 \mathrm{~nm})$ and short (100-200 nm) cylindrical strands with a smooth surface. These poorly ramified strands form long filamentary chains separated from each other. At $35 \mathrm{~V}$, the strands are still forming filamentary chains but they are slightly thicker $(200 \mathrm{~nm})$ and longer $(1 \mu \mathrm{m})$. They also begin to exhibit more branching features. From 40 to $50 \mathrm{~V}$, only highly branched structures are observed. The strands are larger (500 nm to $2 \mu \mathrm{m})$ and longer $(1.5-4 \mu \mathrm{m})$. Their surface is also rougher because of the presence of small nucleation germs. At 55 and $60 \mathrm{~V}$, the strands diameter continues to grow up to $3 \mu \mathrm{m}$ but we observe a decrease in their length (4 to $3 \mu \mathrm{m})$. The strands become rounder and more spherical compared to lower voltages strands. For these voltages, the strands structure is more chaotic due to the multiple-faceted germs presents on the surface.

In Figure 4 representing the evolution of the morphology of gold strands, we can see that gold foams have a similar behavior compared to copper foams. At $25 \mathrm{~V}$ the gold strands are about $100 \mathrm{~nm}$ in width and are $300 \mathrm{~nm}$

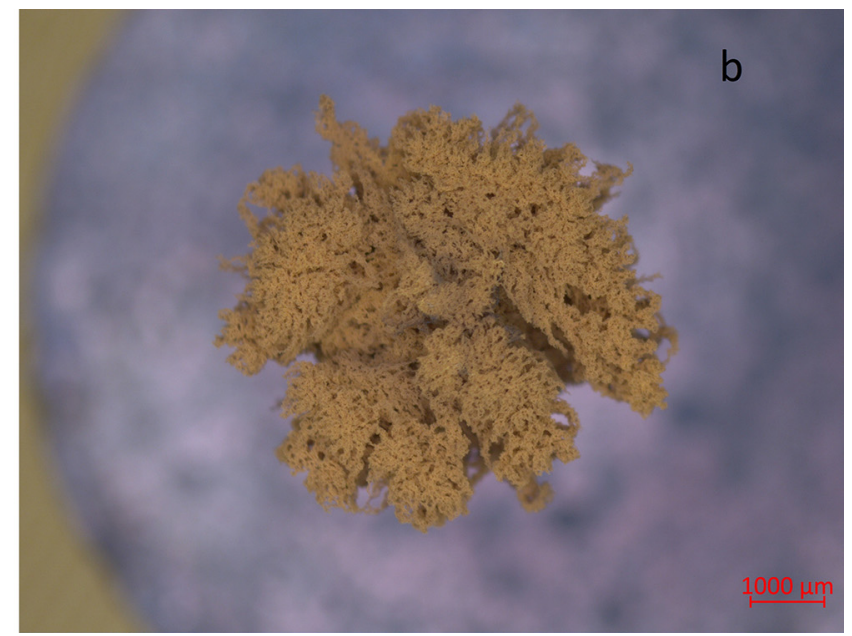

Fig. 2. Picture of metallic foams synthetized by plasma electrolysis deposition: (a) copper; (b) gold. 

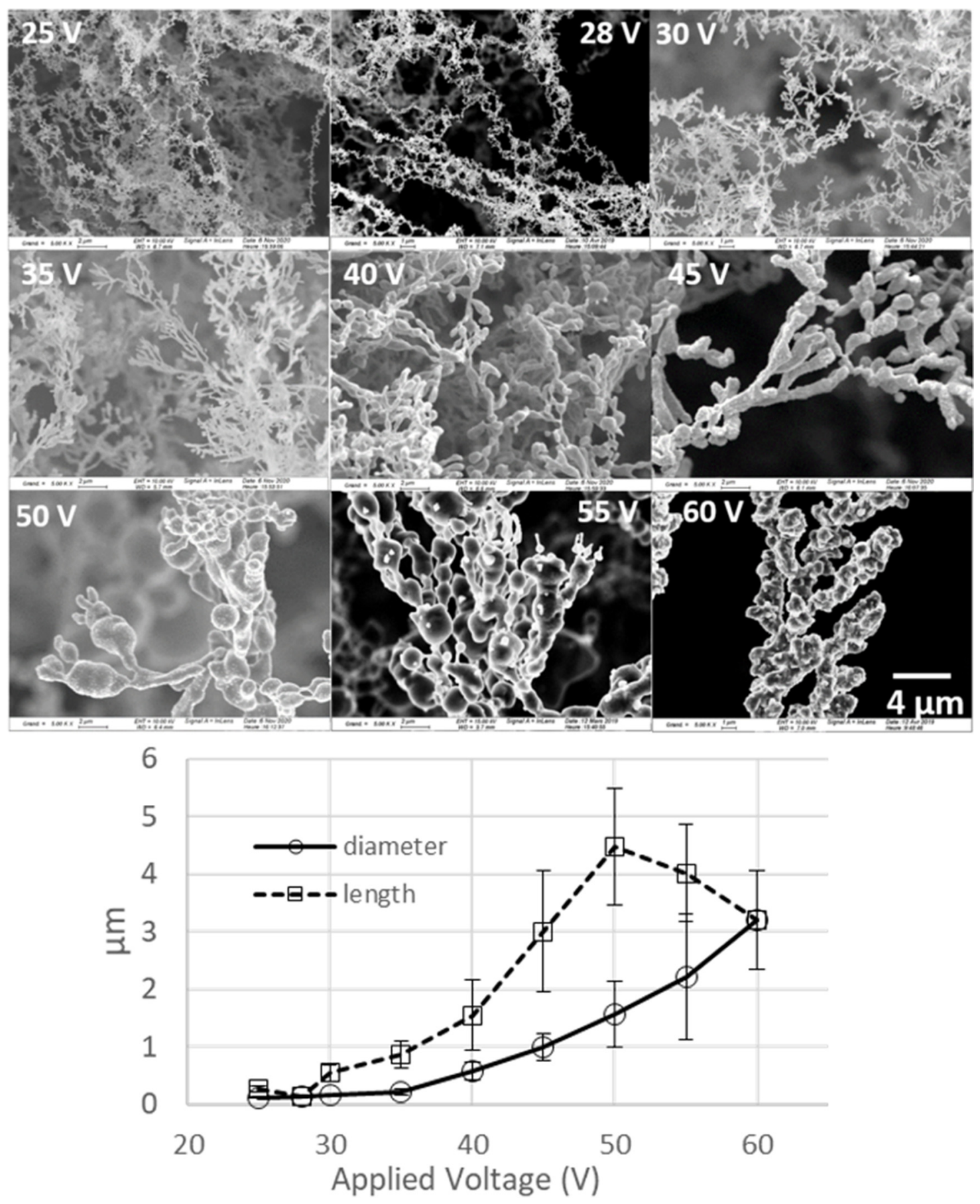

Fig. 3. Evolution of the morphology, diameter and length of copper strands with the voltage.

long. The strands form lengthy filaments with few ramifications. For the foam synthetized at $30 \mathrm{~V}$, we mainly observe bushy and branched structures. The strands are slightly thicker $(300 \mathrm{~nm})$ and longer $(500 \mathrm{~nm})$ and continue to grow as the voltage increases to attain $500 \mathrm{~nm}$ in diameter and $850 \mathrm{~nm}$ in length $(40 \mathrm{~V})$. At $45 \mathrm{~V}$ the morphology of the strands is very different. The foam seems to be formed by an aggregation of spherical strands with some protrusion extending from their surface.
The diameter of theses strands is about $1.7 \mu \mathrm{m}$. At $50 \mathrm{~V}$ the foam is formed by large $(3 \mu \mathrm{m})$ faceted strands and at $55 \mathrm{~V}$ the strands seem fused with each other to create $6.5 \mu \mathrm{m}$ thick hairs. No exploitable sample of gold foams was obtained at $60 \mathrm{~V}$.

For copper foams as well as for gold foams, we can see that the dispersion of diameter and length of the strands is more important as the applied voltage rises (Figs. 3 and 4). 


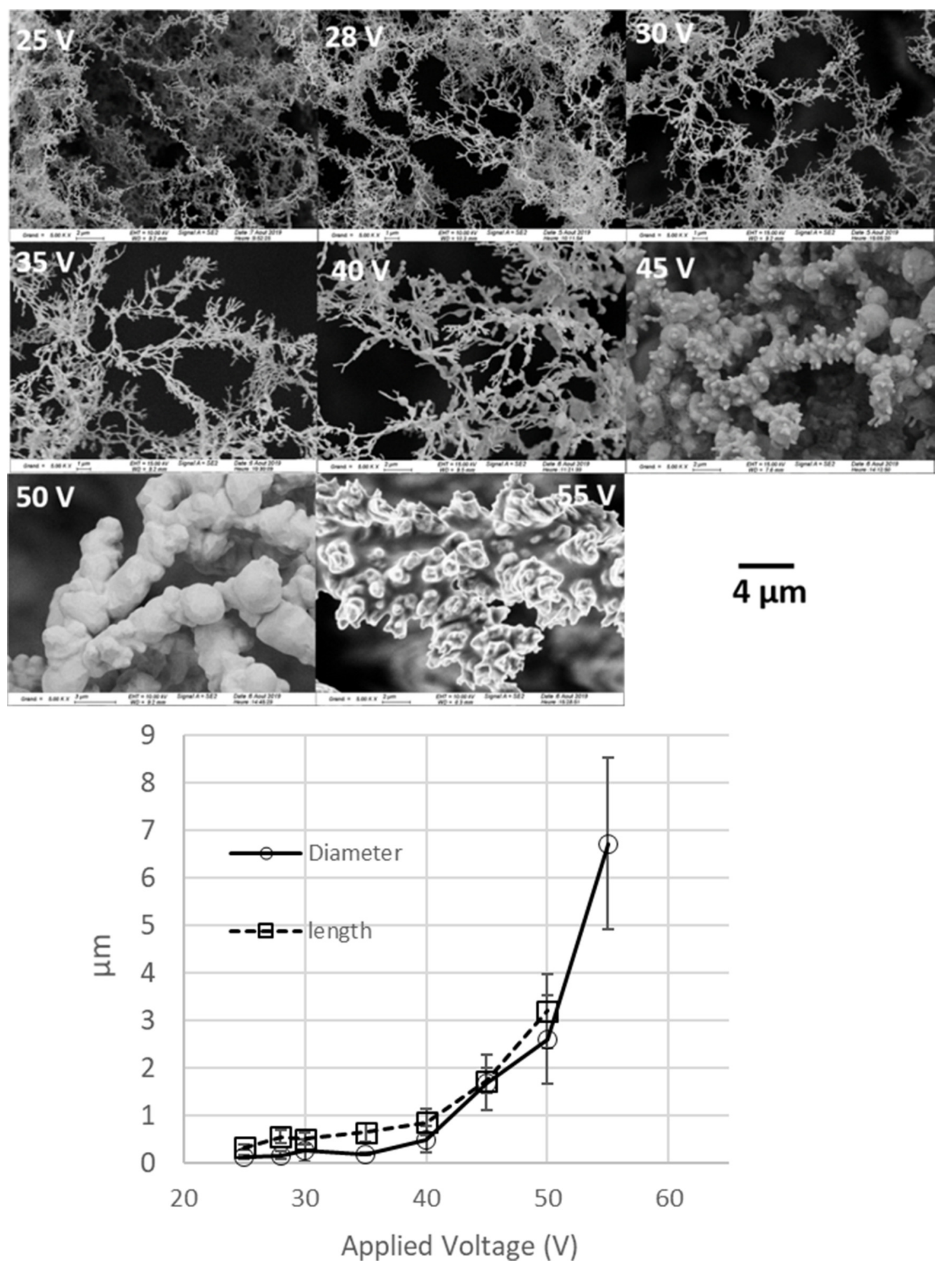

Fig. 4. Evolution of the morphology, diameter and length of gold strands with the voltage. 


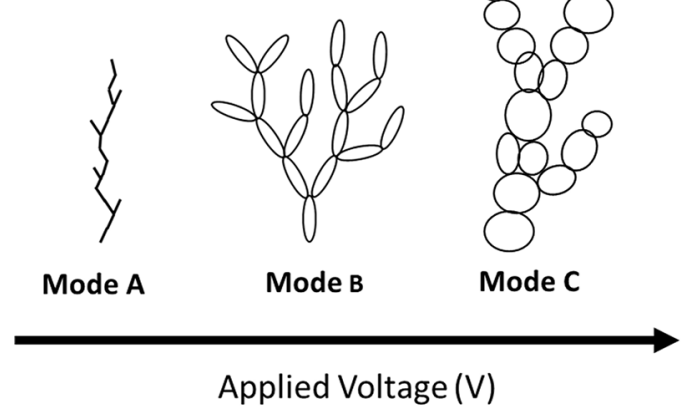

Fig. 5. Growth modes of metallic foams.

\subsection{Growth modes of metallic foams}

From the FE-SEM studies, we can identify three different types (A, B, C) of morphology for the strands as the synthesis voltage increases (Fig. 5). The mode $\mathrm{A}$ is characterized at the microscopic level by thin strands forming long and filamentary chains growing separately. The structure formed can be qualified as "tree-like". The mode B is characterized by elongated and highly branched strands. Because of the multiple branching, the global shape is a "bush like" structure. Finally, the mode C is characterized by almost spherical strands aggregated to each other to form big isolated filaments.

In a previous work [11], we reported that the morphology of the strands was similar to the shape of a cathode discharge in liquid $[12,13]$. This is why we made the hypothesis that the foam grows directly on the plasma discharges created during the process. Here, we show that the three growth behavior identified are very similar to the propagation modes of streamers, a pre-breakdown phenomena observed in plasma discharges in liquids [14,15]. Indeed, streamers can propagate linearly with low branching features as observed for mode A foams. As the voltage increases, the streamers branching becomes more important just like for mode B foams. For higher voltages, the propagation for the streamer is more linear and exhibits few branching features. This behavior corresponds to the structure of mode $\mathrm{C}$ foams. Consistent with our hypothesis, the FE-SEM observations show that the morphology of foams strands probably results from the propagation of plasma streamers in the electrolyte. The augmentation of the strands width and length may be due to an energy increase provided by higher voltages. Indeed, with more energy, the streamers are larger and they can cover more distance during their lifetime. However we do not yet understand how these plasma streamers reduces the metallic ions to make the metallic strands.

\subsection{Microstructure of the strands}

The powder X-ray diffraction patterns of copper and gold (Fig. 6) show that both structures are composed of small crystallites (100-250 nm range with Rietveld's method).
To go further, TEM studies have been conducted to determine the microstructure of the metallic strands. The strands from mode $\mathrm{C}$ foams could not be observed by TEM due to their excessive thickness.

Copper strands (Fig. 7) present a fully crystallized core enveloped by a polycristalline oxide layer. The studies revealed that this layer is composed of $\mathrm{Cu}_{2} \mathrm{O}$ and is about $30 \mathrm{~nm}$ thick (Fig. 7a). For the strand core, the electron diffraction patterns correspond to a monocrystalline face centered cubic (FCC) lattice (Fig. 7b). The lattice parameter is about $3.6 \AA$, which is coherent with bulk copper. The microstructure was the same for all observed strands no matter the applied voltage. Only the mean diameter and length of the strands were increasing with the voltage.

For gold strands (Fig. 8a), we also observe a monocrystalline FCC structure. As observed in Figure $8 \mathrm{~b}$, the lattice does not show any defects such as inclusions, dislocation or holes. The crystalline structure is perfect. Moreover, the observations show that the growth direction of the strands are oriented in accordance to the crystallographic orientations of the lattice. These observations could be an insight in order to determine the conditions of crystallization of the strands resulting from the interaction between the plasma and the electrolyte.

\section{Conclusion}

In this paper we investigated the role of the applied voltage on the morphology and microstructure of copper and gold foams synthetized by plasma electrolysis deposition. As the voltage increases, we observed an augmentation of the foams strands width and length. Between 25 and $60 \mathrm{~V}$, the size of the strands varies between one hundred nanometers to few micrometers. We identified three different type of morphology for the copper strands. The same behavior was observed for gold strands. These results lead to the definition of three growth modes for the metallic foams. The TEM pictures and electron diffraction patterns of metallic strands showed a monocrystalline structure. No lattice defects were observed.

The transmission electron microscope images were recorded at the Institut Jean Lamour in Nancy, France. The authors would like to thanks Dr. J. Ghanbaja from Institut Jean Lamour (Nancy, France) for his contribution to this work.

\section{Author contribution statement}

The experimental work and the writing of the manuscript was done by Julien Pinot. The experimental protocols, the data analysis and the interpretation of the results were performed by Julien Pinot, Ronan Botrel, Frederic Durut and Vincent Vignal. Ludovic Reverdy and Laurent Pescayre contributed to the extensive revising of the manuscript. All authors discussed the results and commented the manuscript. The authors declare no competing financial interests. 


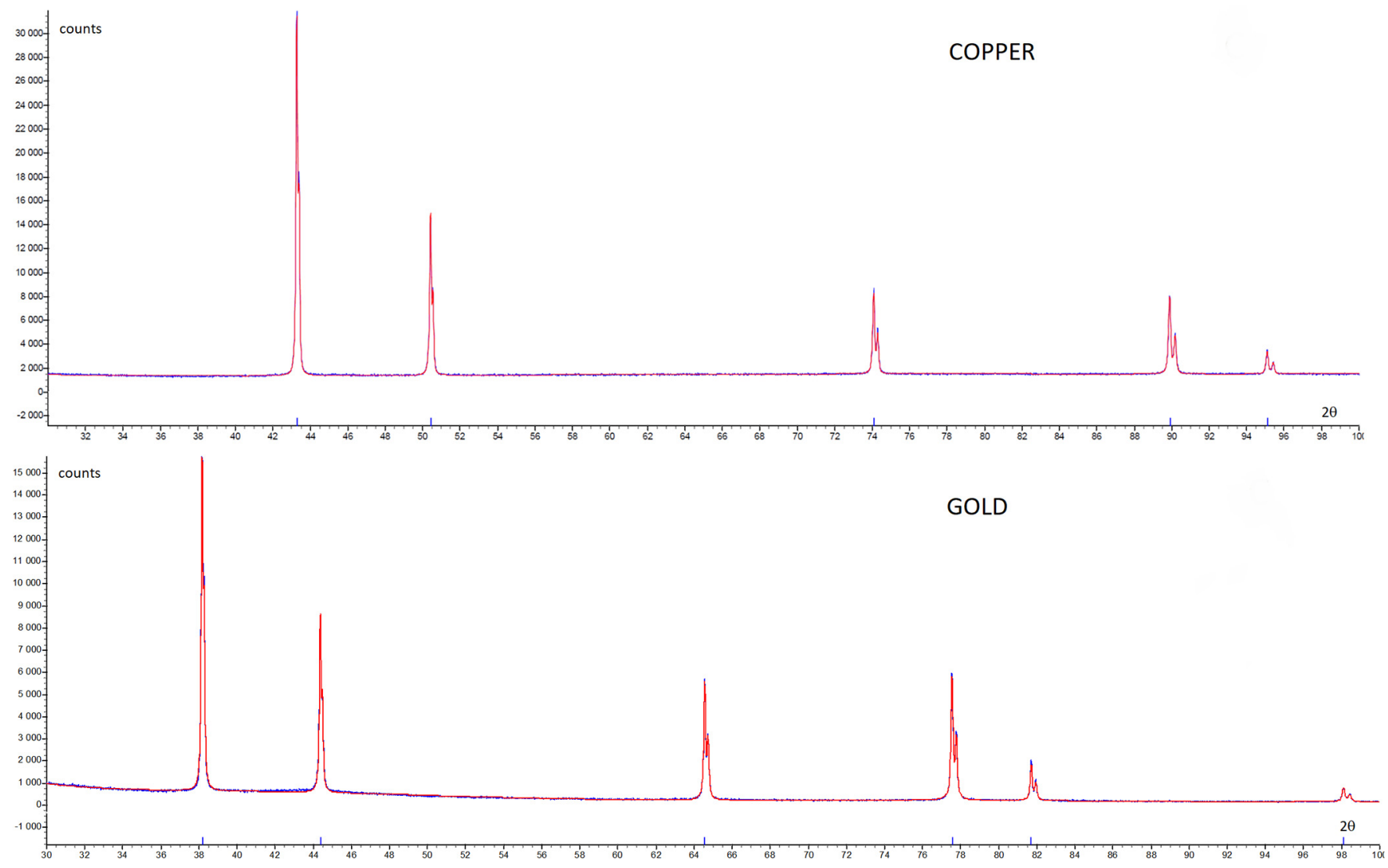

Fig. 6. Powder X-Ray diffraction patterns of copper and gold foams.
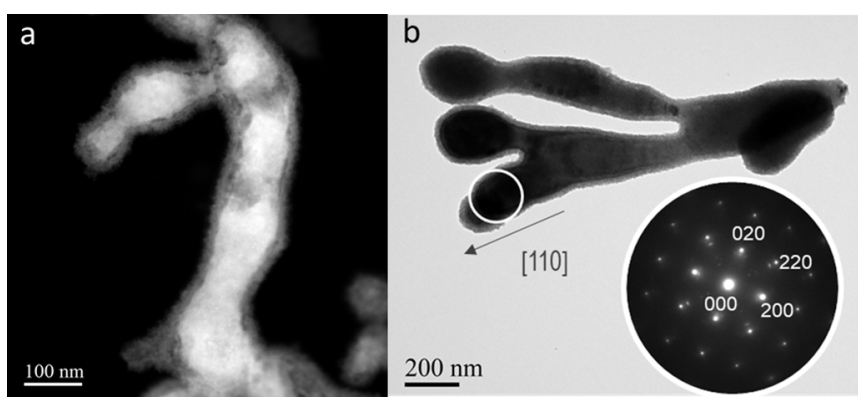

Fig. 7. (a) STEM HAADF picture of a copper strand (28 V). We can clearly see the difference between the strand core composed of bulk copper (white) and the external oxide layer (grey). (b) Copper strands (30V) observed by TEM with the associated electron diffraction pattern.
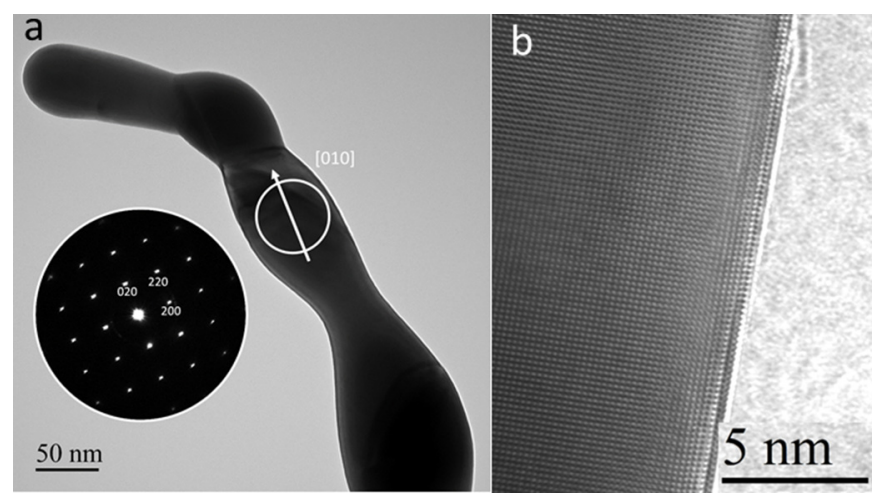

Fig. 8. (a) Gold strand $(30 \mathrm{~V})$ observed by TEM with the associated electron diffraction pattern. (b) Microstructure of a gold strand observed by HRTEM. 


\section{References}

1. J.G. Moreau, Ph.D Thesis, University of Bordeaux, 2018

2. P.S. Liu, G.F. Chen, Porous Materials Processing and Applications (Elsevier, Tsinghua, 2014)

3. B. Tappan et al., Angew. Chem. Int. Ed. 49, 4544 (2010)

4. J. Banhart, Adv. Eng. Mater. 15, 3 (2013)

5. F. Baumgärtner et al., in Metal Matrix Composites and Metallic Foams, edited by T.W. Clyne and F.S. Simancik (Wiley, Weinheim, 2000)

6. C. Körner et al., in Metal Matrix Composites and Metallic Foams, edited by T.W. Clyne and F.S. Simancik (Wiley, Weinheim, 2000)

7. S.H. Kim et al., ACS Appl. Mater. Interfaces 8, 34706 (2016)

8. B. Tappan et al., J. Am. Chem. Soc. 128, 6589 (2006)
9. F. Qian et al., Nano Lett. 20, 131 (2020)

10. R. Botrel, EP 2824219 B1, EU patent (2015)

11. S. Rocher et al., Eur. Phys. J. Appl. Phys. 81, 10803 (2018)

12. P. Ceccato, Ph.D. thesis, Paris-Saclay University, 2010

13. P.J. Bruggeman et al., Plasma Sources Sci. Technol. 25, $053002(2016)$

14. R. Hebner, in The Liquid State and its Electrical Properties, NATO ASI Ser., Ser. B. 193, 519 (1987)

15. O. Lesaint, J. Phys. D: Appl. Phys. 49, 144001 (2016)

Open Access This article is distributed under the terms of the Creative Commons Attribution License https://creativecom mons.org/licenses/by/4.0 which permits unrestricted use, distribution, and reproduction in any medium, provided the original author(s) and source are credited.

Cite this article as: Julien Pinot, Ronan Botrel, Frederic Durut, Ludovic Reverdy, Laurent Pescayre and Vincent Vignal, Morphology of metallic foams synthetized by plasma electrolysis deposition, Eur. Phys. J. Appl. Phys. 97, 2 (2022) 